\title{
Implementation and performance analysis of Video Edge Detection system on Multiprocessor Platform
}

\author{
Mandeep Kaur \\ Department of Electronics and Communication \\ Thapar University \\ Patiala, Punjab, India
}

\author{
Kulbir Singh \\ Department of Electronics and Communication \\ Thapar University \\ Patiala, Punjab, India
}

\begin{abstract}
This paper presents an agile development, implementation of Edge Detection on SMT8039 based Video And Imaging module. With the development of video processing techniques its algorithm becomes more complicated. High resolution and real time application cannot be implemented with single CPU or DSP. The system offers significant performance increase over current programmable DSP-based implementations. This paper shows that the considerable performance improvement using the FPGA solution results from the availability of high $\mathrm{I} / \mathrm{O}$ resources and pipelined architecture. FPGA technology provides an alternative way to obtain high performance. Prototyping a design with FPGA offer some advantages such as relatively low cost, reduce time to market, flexibility. Another capability of FPGA is the amount of support of logic to implement complete systems/subsystems and provide reconfigurable logic for purpose of application specific based programming. DSP's to provide more and more power and design nearly any function in a large enough FPGA, this is not usually the easiest, cheapest approach. This paper designed and implemented an Edge detection method based on coordinated DSP-FPGA techniques. The whole processing task divided between DSP and FPGA. DSP is dedicated for data $I / O$ functions. FPGA's task is to take input video from DSP to implement logic and after processing it gives back to DSP. The PSNR values of the all the edge detection techniques are compared. When the system is validated, it is observed that Laplacian of Gaussian method appears to be the most sensitive even in low levels of noise, while the Robert, Canny and Prewitt methods appear to be barely perturbed. However, Sobel performs best with median filter in the presence of Gaussian, Salt and Pepper, Speckle noise in video signal.
\end{abstract}

Keywords-Multiprocessor platform; Edge detection; Performance evaluation; noise.

\section{INTRODUCTION}

Video processing has been used in many fields such as industry, military, medical image processing, surveillances recording etc. Video and imaging applications demand a range of processes to be performed in single applications. Edge detection is one of the basic characteristics of the image [1]. It is an important basis for the field of image analysis such as: the image segmentation, target area identification, extraction and other regional forms .It is widely used in image segmentation, image recognition, and texture analysis of them. Edge detection[2] technology must not only detect the image gray value of the non-continuity, but also to determine their exact location .Although you can use multiple DSP's to provide more and more power and design nearly any function in a large enough FPGA, this is not usually the easiest, cheapest approach[3]-[6]. The obvious result is to mix the two technologies benefits of co-processing. But DSP and FPGA designs are quite disparate disciplines, involving very different techniques, skills and tools [7][8]. But the differences in DSP and FPGA create obstacles to a fluid co-design process rather unpalatable to a specialist in one of the two fields and even more so to an expert of neither. Integrating the hardware [8][14] it also presents a significant amount of work that you could avoid if you stuck with just one technology.

\section{EDGE DETECTION}

Edge has two properties--the direction and the magnitude [1], [2]. Usually the change of the gray level along the edge is flat, but the pixels perpendicular to the edge change dramatically. According to the characteristics of intensity change, it can be divided into step-type and roof- type. In step type, both sides of the pixel in value have changed significantly, and roof type, it is located in the gray scale to reduce the rate of change from the turning point. This paper introduces edge detection for video [10]-[16] on DSP-FPGA system i.e. SMT8039. These algorithms are based on the detection of discontinuities in the values of the grey levels of the image. The most widely used techniques are the generation of a differential image by means of Sobel, Prewitt, Robert, Canny and LOG operator[17]-[21]. The characteristics of these operators, regularity and efficiency, make them adequate for its implementation in an application specific architecture. These operators [5] are based on the differential approach to edge detection. With this approach, a differential image $G$ is generated from the original image $\mathrm{F}$, where the changes in grey levels are accentuated. After this, the edges are detected [10][13] by means of the comparison of the amplitude values to a predefined threshold level. These are based on the gradient operator. The first derivative of the digital image is based on various approximations to the 2-D gradient. The gradient of the image $f(x, y)$ at location $(x, y)$ is defined as the vector.

$$
\Delta f=\left\lfloor\begin{array}{l}
\partial f / \partial x \\
\partial f / \partial y
\end{array}\right\rfloor
$$

We know that gradient vector points in the
direction of maximum rate of change of at


coordinates $(\mathrm{x}, \mathrm{y})$. An important quantity in edge detection is magnitude of this vector. $\Delta \mathrm{f}=\operatorname{mag}(\Delta \mathrm{f})=\left[\mathrm{G}_{\mathrm{x}}{ }^{2}+\mathrm{G}_{\mathrm{y}}{ }^{2}\right]^{1 / 2}$.The direction of the gradient vector also is an important quantity. Let $\quad \alpha(x, y)$ represents the direction [1],[2] angle of the vector $\Delta \mathrm{f}$ at $(\mathrm{x}, \mathrm{y})$ then from vector analysis:

$$
\alpha(x, y)=\tan ^{-1}\left(\frac{G y}{G x}\right)
$$

Computational of the gradient of an image is based on obtaining the partial derivatives $\partial \mathrm{f} / \partial \mathrm{x}$ and $\partial \mathrm{f} / \partial \mathrm{y}$ at every pixel location. The $3 \mathrm{X} 3$ area mask in Fig. 1 for Sobel in Fig. 2 and Prewitt in Fig. 3 operations mask of $3 \times 3$, and for Robert operation 2X2 mask is shown in Fig. 4 are used to convolve with each pixel values of the image

\begin{tabular}{|c|c|c|}
\hline$Z_{1}$ & $Z_{2}$ & $Z_{3}$ \\
\hline$Z_{4}$ & $Z_{5}$ & $Z_{6}$ \\
\hline$Z_{7}$ & $Z_{8}$ & $Z_{9}$ \\
\hline
\end{tabular}

Figure $1.3 \times 3$ neighboring of pixels in an image

\begin{tabular}{|c|c|c|}
\hline-1 & -2 & -1 \\
\hline 0 & 0 & 0 \\
\hline 1 & 2 & 1 \\
\hline
\end{tabular}

\begin{tabular}{|l|l|l|}
\hline-1 & 0 & 1 \\
\hline-2 & 0 & 2 \\
\hline-1 & 0 & 1 \\
\hline
\end{tabular}

Figure 2. Sobel matrix $\mathrm{x}$ and $\mathrm{y}$ directional

\begin{tabular}{|c|c|c|}
\hline-1 & -1 & -1 \\
\hline 0 & 0 & 0 \\
\hline 1 & 1 & 1 \\
\hline
\end{tabular}

\begin{tabular}{|c|c|c|}
\hline-1 & -1 & -1 \\
\hline 0 & 0 & 0 \\
\hline 1 & 1 & 1 \\
\hline
\end{tabular}

Figure 3.Prewitt matrix $\mathrm{x}$ and $\mathrm{y}$ directional

\begin{tabular}{|c|c|}
\hline-1 & 0 \\
\hline 0 & 1 \\
\hline
\end{tabular}

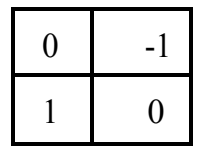

Figure 4.Robert matrix $\mathrm{x}$ and $\mathrm{y}$ directional

For Sobel equation is

$$
\begin{gathered}
\mathrm{Gx}=(\mathrm{Z7}+2 \mathrm{Z8}+\mathrm{Z9})-(\mathrm{Z} 1+2 \mathrm{Z} 2+\mathrm{Z3}) \\
G y=(Z 3+2 Z 6+Z 9)-Z 1+2 Z 4+Z 7)
\end{gathered}
$$

For prewitt equation is

$$
\begin{gathered}
G x=(Z 7+Z 8+Z 9)-(Z 1+Z 2+Z 3) G y \\
=(Z 3+Z 6+Z 9)-(Z 1+Z 4 \\
+Z 7)
\end{gathered}
$$

$$
\mathrm{Gx}=(\mathrm{Z} 1-\mathrm{Z} 4)
$$

$$
G y=(Z 2-Z 3)
$$

In this formulation, the difference between the first and third rows of the $3 \times 3$ image region approximates the derivative in $\mathrm{x}$-direction and the difference between the third and first columns approximates the derivatives in the y-direction. However this implementation is not always desirable because of the computational burden required by Squares and Square root. The equation is much more attractive computationally, and it still preserves relative changes in gray levels. The laplacian is not used in original form because its magnitude produces double edges. The purpose of this operator is to provide image with zero crossing used to establish the location of edges.

\section{SYSTEM ARCHITECTURE}

System architecture includes:

1. CCD camera for PAL or NTSC standard video input.

2. TMS320DM642 DSP board is used as executing image processing algorithms [4].

3. Video processing board is shown as dashed frame in Fig. 6. FPGA is used as logic unit. Virtex 4 FPGA is connected to the DSP's EMIF[4][5]. This allows high speed transfers to be initiated at request. The Module features a single Philips Semiconductors SAA7109AE/108AE video decoder/encoder that accept most PAL and NTSC standards, and can output processed images in PAL/NTSC or VGA $(1280 \times 1024$, or HDTV Y/Cb/Cr) The DM642 has 128 Mbytes of high speed SDRAM (Micron MT48LC64M32F2S5) available onboard for image processing and an $8 \mathrm{Mbytes}$ FLASH device is fitted to store programs and FPGA configuration information. The function SAA7109AE/108AE is to change analog video signals from $\mathrm{CCD}$ into digital signal and the image data with the format of YUV 4:2:2 are stored in SDRAM.

4. VGA display is used as displaying output Image.

\section{DESIGN AND IMPLEMENTATION OF EdGE DETECTION SYSTEM ON SOFTWARE}

The software 3L Diamond for SMT339 provides a model describing multiprocessor system as a no. of independent tasks that communicate together over a channel[4][5]. Weather these tasks are executing on DSP or FPGA Diamond manages the interconnection and programming so that you can concentrate on the application In this system, different module (tasks) are created. These connections are logically defined for communication between different tasks for DSP and FPGA [14][15][21]. In DSP, a Task Dsp_pal which is written in c language, In DSP, frames information like no. of input frames, no. of output frame, video memory [1] for channel A, B video capture registers, FIFO registers are defined in the library are imported, there are 3 video ports: Vp1 is used for input the video from the camera, $\mathrm{Vp} 2$ is undefined and video port $\mathrm{Vp} 0$ is used for displaying video on VGA display. For RGB656 format this involves a single EDMA channel, so DMA transfer 64 bit data and for $\mathrm{YCbCr}$, it contains 3 separate channels for initialization.

And for Robert equation is 


\section{Video EdGE Detection System Tests}

In this paper we take a frame of video and we perform different edge detection techniques on this frame. In this we added noise like Gaussian, salt and pepper, speckle[22]-[25]. In this paper, we estimate the effect of noise on different edge detection algorithms that which one is more sensitive to the noise, Original video frame is shown in Fig. 10. Fig. 11 shows effects of noise on different edge detection techniques at different PSNR values.

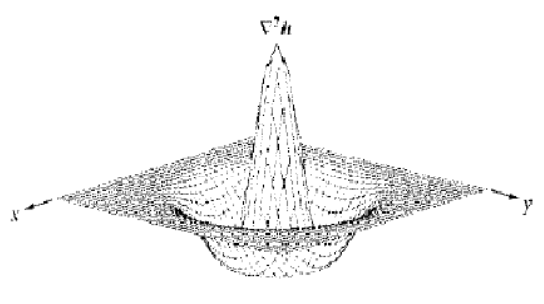

(a)

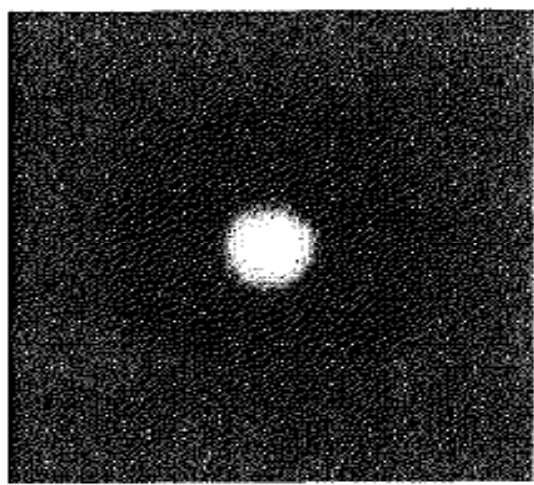

(b)

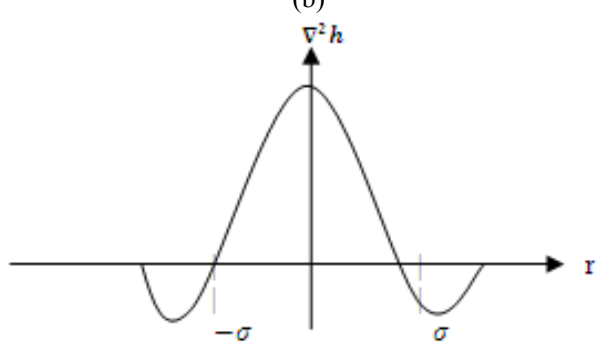

(c)

\begin{tabular}{|c|c|c|c|c|}
\hline 0 & 0 & -1 & 0 & 0 \\
\hline 0 & -1 & -2 & -1 & 0 \\
\hline-1 & -2 & 16 & -2 & -1 \\
\hline 0 & -1 & -2 & -1 & 0 \\
\hline 0 & 0 & -1 & 0 & 0 \\
\hline
\end{tabular}

(d)

Figure 5.Laplacian of Gaussian) 3D- plot (a) Image black is negative, gray is zero and white is positive. (c) Cross section showing zero crossing (d) $5 \times 5$ mask of LOG.

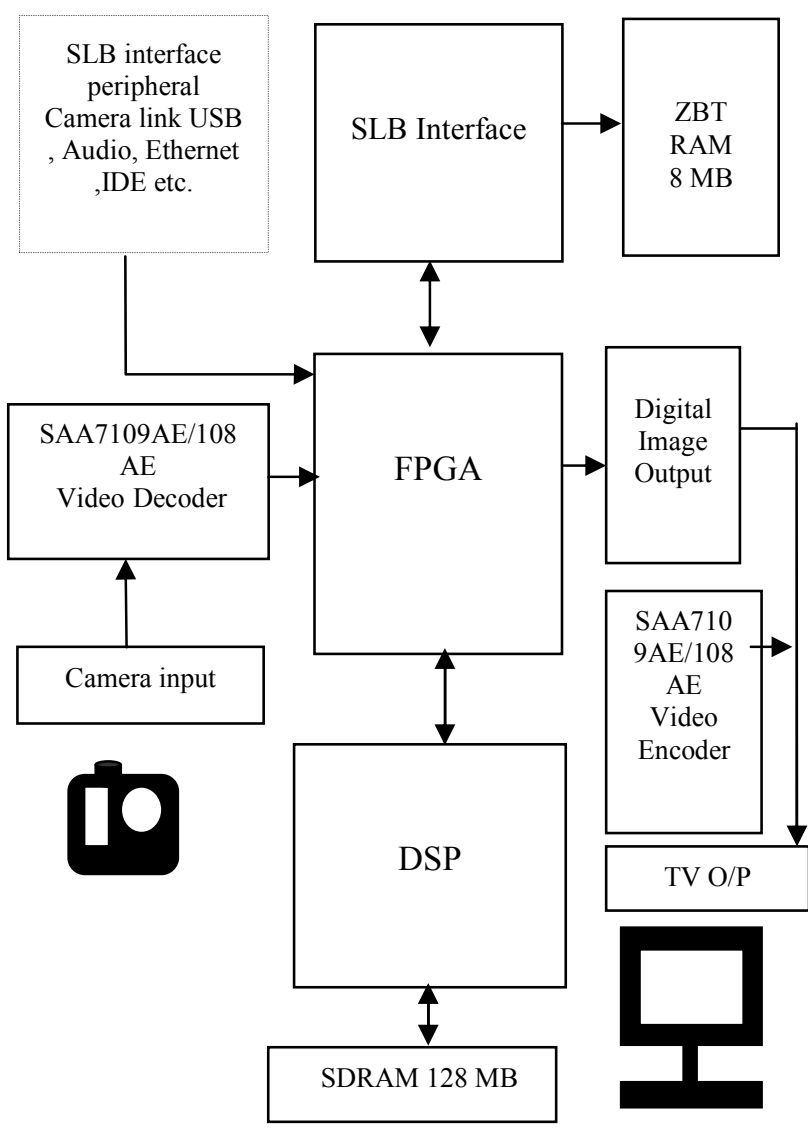

Figure 6. System Architecture

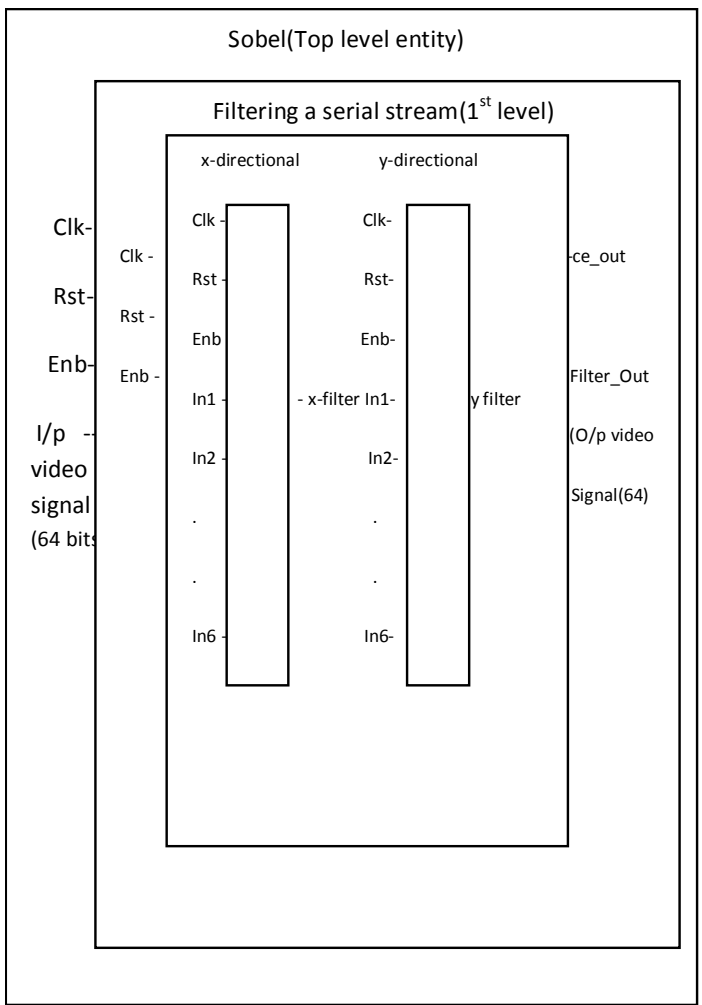

Figure 7.Entity for edge detection (Sobel, Prewitt) 


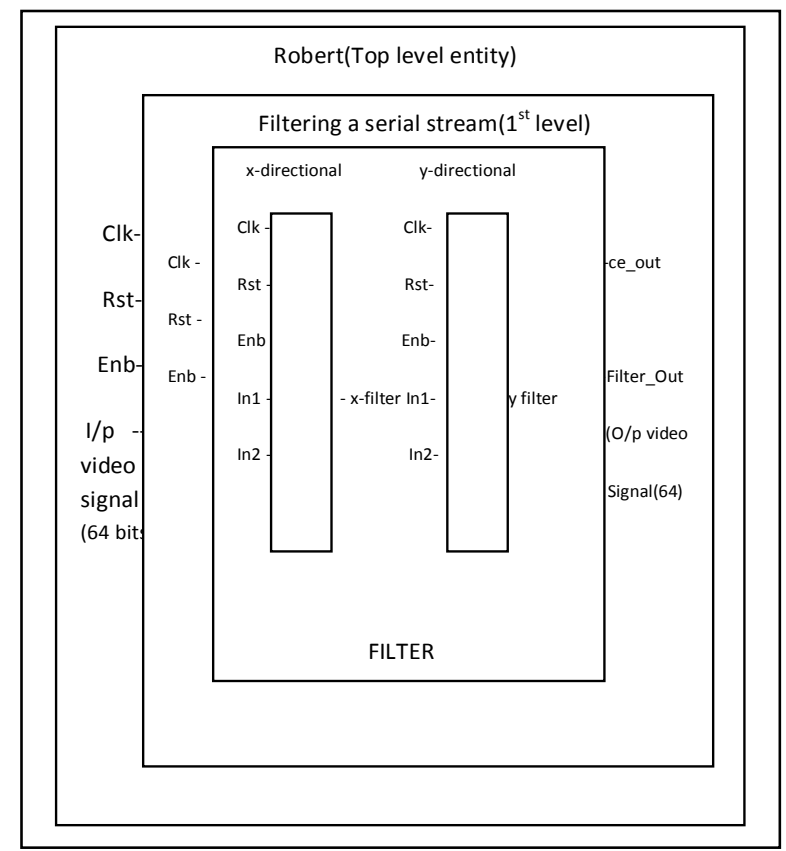

Figure 8.Entity for edge detection (Roberts)

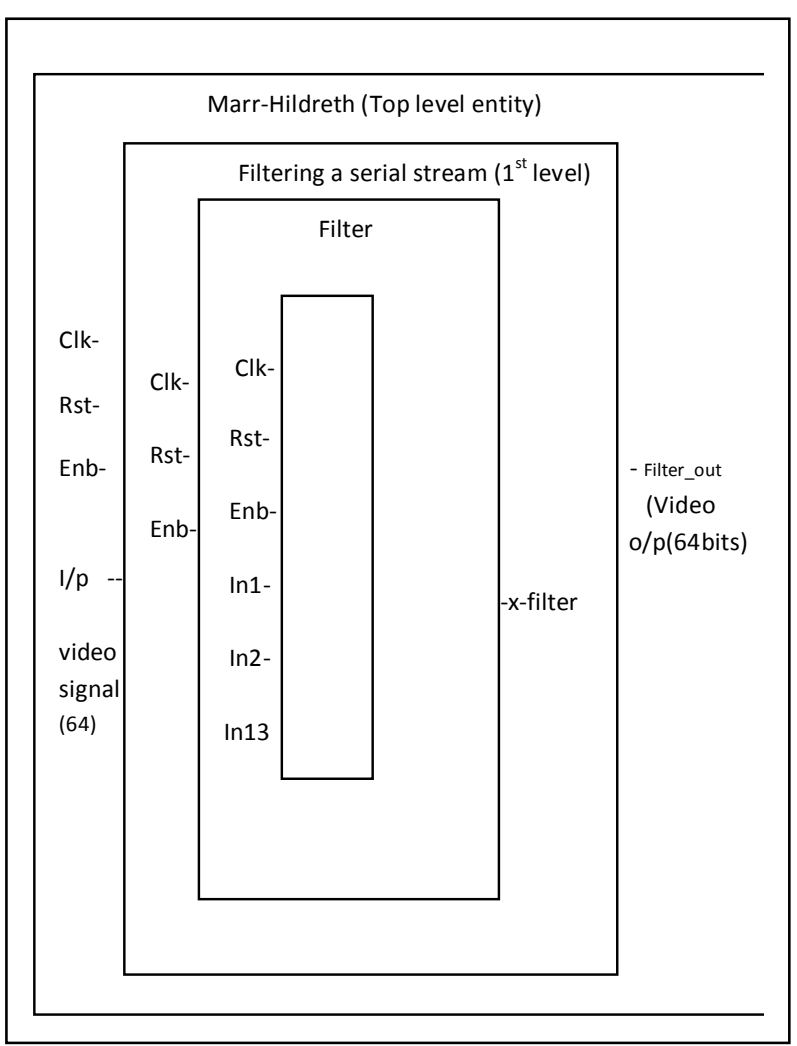

Figure 9.Entity for edge detection (Marr -Hildreth)s

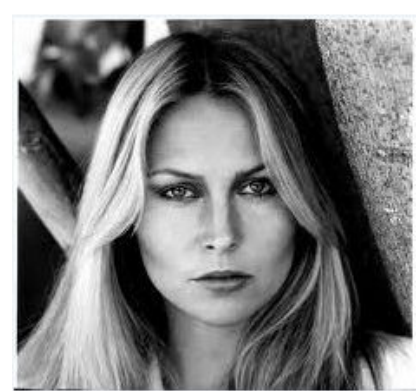

Figure 10.Original Frame

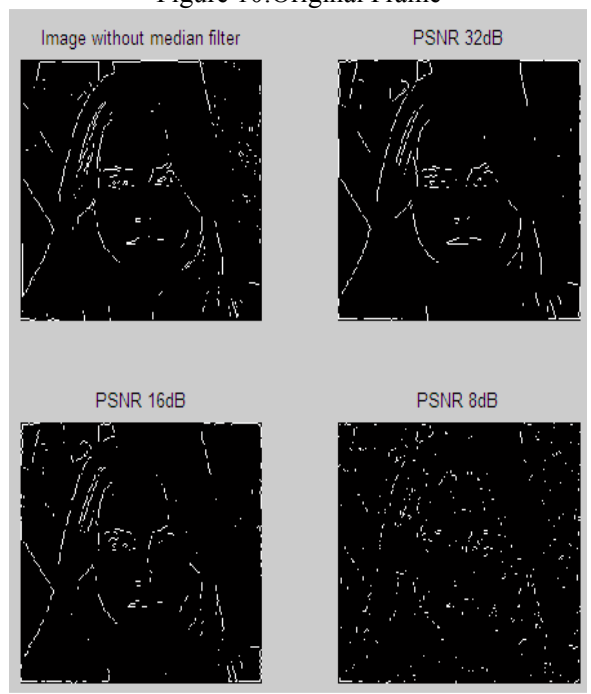

(a)

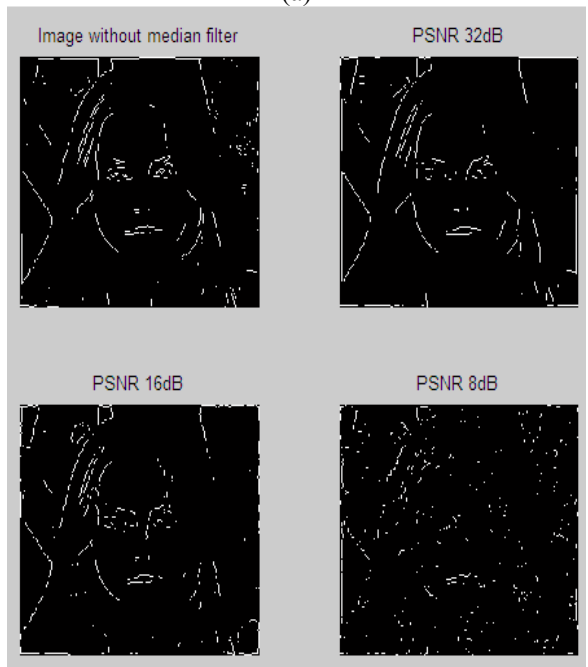

(b) 


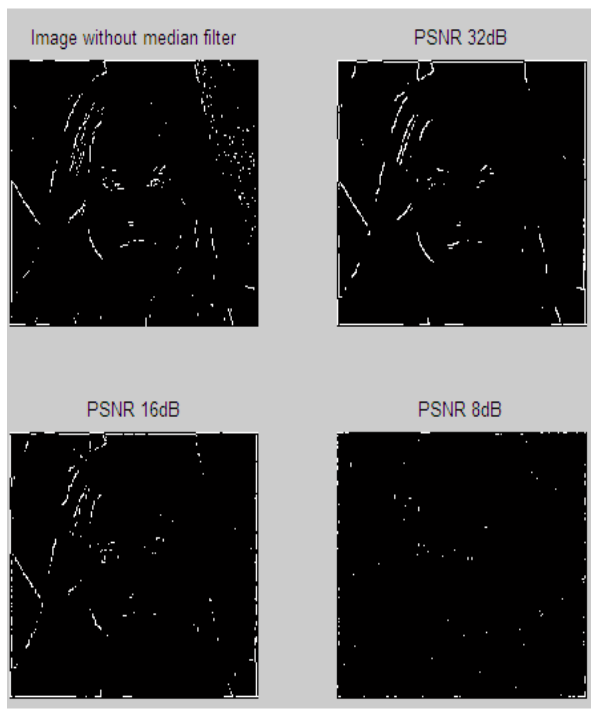

(c)

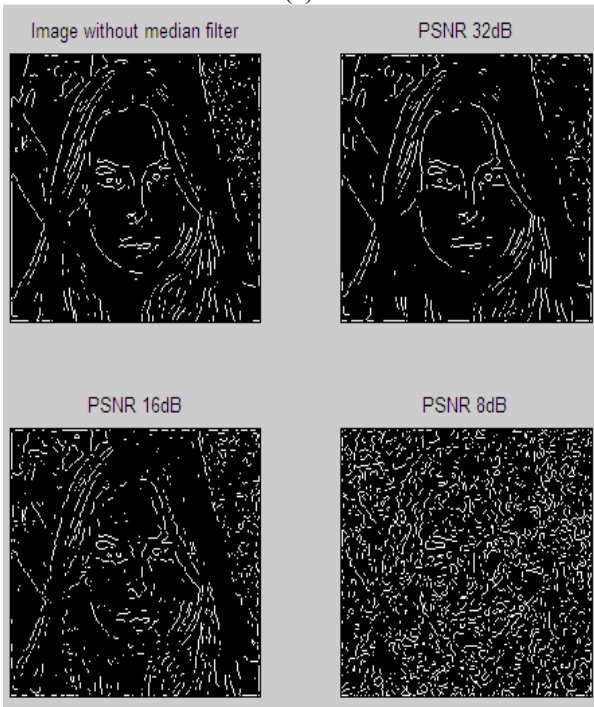

(d)

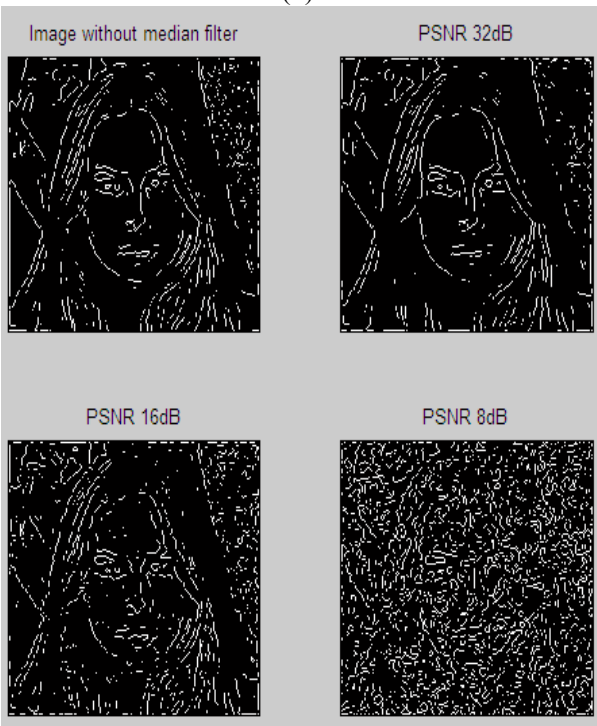

(e)
Figure 11. (a) Sobel operation at different PSNR (b) Prewitt operation at different PSNR (c) Roberts operation at different PSNR (d) Marr-Hildreth operation at different PSNR (e) Canny operation at different PSNR

TABLE I.

\begin{tabular}{|c|c|c|c|c|}
\hline \multicolumn{5}{|c|}{$\begin{array}{c}\text { EFFECT OF GAUSSIAN NOISE ON DIFFERENT EDGE } \\
\text { DETECTION TECHNIQUES }\end{array}$} \\
\hline $\begin{array}{c}\text { PSNR } \\
\text { of } \\
\text { noisy } \\
\text { image }(d B)\end{array}$ & $\begin{array}{c}\text { PSNR } \\
\text { of noisy } \\
\text { image } \\
\text { after } \\
\text { median } \\
\text { filter(dB) }\end{array}$ & $\begin{array}{c}\text { Edge } \\
\text { Detection } \\
\text { Technique } \\
\text { (dB) }\end{array}$ & $\begin{array}{c}\text { PSNR of } \\
\text { edge } \\
\text { detection } \\
\text { before } \\
\text { Median } \\
\text { filter(dB) }\end{array}$ & $\begin{array}{c}\text { PSNR } \\
\text { Of } \\
\text { edge } \\
\text { detection } \\
\text { after } \\
\text { Median } \\
\text { filter(dB) }\end{array}$ \\
\hline \multirow{5}{*}{38.3437} & \multirow{5}{*}{24.4731} & Sobel & 3.1254 & 4.1513 \\
\hline & & Prewitt & 11.0582 & 12.058 \\
\hline & & Robert & 12.6772 & 11.0199 \\
\hline & & $\begin{array}{l}\text { Marr- } \\
\text { Hildreth }\end{array}$ & 10.7655 & 10.2409 \\
\hline & & Canny & 10.3832 & 11.2856 \\
\hline \multirow{5}{*}{16.0414} & \multirow{5}{*}{21.4583} & Sobel & 7.1792 & 13.0138 \\
\hline & & Prewitt & 8.6966 & 13.1051 \\
\hline & & Robert & 15.1037 & 12.8259 \\
\hline & & $\begin{array}{c}\text { Marr- } \\
\text { Hildreth }\end{array}$ & 10.9128 & 9.88 \\
\hline & & Canny & 4.9631 & 11.2809 \\
\hline \multirow{5}{*}{10.487} & \multirow{5}{*}{17.297} & Sobel & 0.0508 & 12.7839 \\
\hline & & Prewitt & 9.6073 & 13.2758 \\
\hline & & Robert & 15.5064 & 13.772 \\
\hline & & $\begin{array}{c}\text { Marr- } \\
\text { Hildreth }\end{array}$ & 10.7196 & 10.0412 \\
\hline & & Canny & 9.9918 & 11.1472 \\
\hline \multirow{5}{*}{8.1645} & \multirow{5}{*}{14.7276} & Sobel & -3.857 & 11.6824 \\
\hline & & Prewitt & -3.5126 & 11.5007 \\
\hline & & Robert & 13.2864 & 11.2624 \\
\hline & & $\begin{array}{c}\text { Marr- } \\
\text { Hildreth }\end{array}$ & 10.404 & 10.2732 \\
\hline & & Canny & 10.2075 & 10.8719 \\
\hline
\end{tabular}

We also compare the PSNR values of the all the edge detection techniques which are listed above with different kinds of noise levels and noise type [21] [26] [27]. Out of five operators, Sobel edge detection method is found as the best in detecting the edges in noisy images. By applying median filter to the noisy image, noise is removed from the images and then all techniques are applied to filtered frame [28]. So the paper concludes that Sobel edge detector with the Median filter performs well in detecting the edges, when compared to other edge detector with median filter [28][23].

In Fig. 11a, shows Sobel operation, Fig. 11b shows Prewitt operation, Fig 11c shows Robert's operation and Fig. 11d shows LOG operation and Fig. 11e shows Canny. Fig 11a shows image with median filter original and second image at $32 \mathrm{~dB}$ PSNR, $3^{\text {rd }}$ at $16 \mathrm{~dB}$ and last one at $8 \mathrm{~dB}$. Here PSNR is 
calculated by comparing the mean of the pixel values with the mean of the additive Gaussian noise [29][23]. The noise is multiplied by the proper scale so that it has a mean value of 0.016 for the $32 \mathrm{~dB}$ case. At this PSNR level, all methods return acceptable results. As the values of PSNR decreased, performance decreased [30]-[35].

TABLE II.

\begin{tabular}{|c|c|c|c|c|}
\hline \multicolumn{5}{|c|}{ EFFECT OF SALT \& PEPPER NOISE ON DIFFERENT } \\
EDGE DETECTION TECHNIQUES
\end{tabular}

TABLE III.

\begin{tabular}{|c|c|c|c|c|}
\hline \multicolumn{5}{|c|}{$\begin{array}{c}\text { EFFECT OF SPECKLE NOISE ON DIFFERENT EDGE } \\
\text { DETECTION TECHNIQUES }\end{array}$} \\
\hline $\begin{array}{c}\text { PSNR } \\
\text { Of } \\
\text { noisy } \\
\text { image }(d B)\end{array}$ & $\begin{array}{c}\text { PSNR } \\
\text { Of } \\
\text { Noisy } \\
\text { Image } \\
\text { After } \\
\text { Median } \\
\text { Filter(dB) }\end{array}$ & $\begin{array}{c}\text { Edge } \\
\text { Detection } \\
\text { Techniques } \\
\text { (dB) }\end{array}$ & $\begin{array}{c}\text { PSNR } \\
\text { of edge } \\
\text { detection } \\
\text { before } \\
\text { Median } \\
\text { filter } \\
\text { (dB) }\end{array}$ & $\begin{array}{l}\text { PSNR edge } \\
\text { detection } \\
\text { after } \\
\text { Median } \\
\text { filter } \\
\text { (dB) }\end{array}$ \\
\hline \multirow[t]{2}{*}{23.9577} & \multirow[t]{2}{*}{23.417} & Sobel & 3.3957 & 4.332 \\
\hline & & Prewitt & 12.4686 & 12.6079 \\
\hline
\end{tabular}

\begin{tabular}{|c|c|c|c|c|}
\hline & & Robert & 14.3418 & 12.563 \\
\hline & & $\begin{array}{c}\text { Marr- } \\
\text { Hildreth }\end{array}$ & 10.3874 & 11.3048 \\
\hline & & Canny & 10.2656 & 10.78 \\
\hline \multirow{5}{*}{21.0908} & \multirow{5}{*}{23.3807} & Sobel & 10.6997 & 13.1004 \\
\hline & & Prewitt & 12.7342 & 13.143 \\
\hline & & Robert & 15.2956 & 12.563 \\
\hline & & $\begin{array}{c}\text { Marr- } \\
\text { Hildreth }\end{array}$ & 10.3882 & 11.3595 \\
\hline & & Canny & 10.2664 & 10.834 \\
\hline \multirow{5}{*}{15.02323} & \multirow{5}{*}{20.8912} & Sobel & 7.9304 & 13.7925 \\
\hline & & Prewitt & 12.7663 & 13.0254 \\
\hline & & Robert & 18.2607 & 13.2232 \\
\hline & & $\begin{array}{c}\text { Marr- } \\
\text { Hildreth }\end{array}$ & 10.3815 & 11.704 \\
\hline & & Canny & 10.2504 & 11.3389 \\
\hline \multirow{5}{*}{18.2588} & \multirow{5}{*}{22.5847} & Sobel & 10.1194 & 13.438 \\
\hline & & Prewitt & 12.1019 & 13.8302 \\
\hline & & Robert & 16.5047 & 12.7849 \\
\hline & & $\begin{array}{c}\text { Marr- } \\
\text { Hildreth }\end{array}$ & 10.3638 & 11.4982 \\
\hline & & Canny & 10.2387 & 11.0434 \\
\hline
\end{tabular}

TABLE IV

RESOURCES USED

\begin{tabular}{|l|l|l|l|}
\hline BUFG & DCM & RAM16 & SLICES \\
\hline 4 out of 32 & 2 out of 12 & 1 out of 232 & $\mathbf{9 5 3}$ out of 25280 \\
\hline $12 \%$ & $16 \%$ & $1 \%$ & $13 \%$ \\
\hline
\end{tabular}

TOTAL TIME FOR BUILDING THE APPLICATION:

Synthesis time: $\quad 70$ second $(1 \mathrm{~min}$ and $10 \mathrm{sec})$

Translate: $\quad 19$ second $(0 \mathrm{~min}$ and $19 \mathrm{sec})$

Map: $\quad 60$ second $(1 \mathrm{~min}$ and $10 \mathrm{sec})$

Place and route: $\quad 61 \mathrm{~second}(1 \mathrm{~min}$ and $1 \mathrm{sec})$

Generate bit stream: 32 second ( $0 \mathrm{~min}$ and $32 \mathrm{sec})$

After the verification of this design in Xilinx FPGA development board, video edge detection system achieves the desired test results. Both filtering and edge detection perform well.

\section{CONCLUSION AND FUTURE WORK}

This paper realizes a DSP-FPGA based video edge detection system and combines the respective strengths of FPGA's and DSP's can be starting with a scalable system. Supported by a comprehensive software environment, such complex hardware can become both adaptable and accessible Verification on SMT8039 development board and on VGA display indicates that the system can accurately detect the images edge and satisfy requirements of the real-time video image edge detection. Finally it achieves the desired experimental results. Out of five operators, Sobel edge detection method is found to be the best in detecting the edges in noisy images. The Laplacian of Gaussian method appears to be the most sensitive to even low levels of noise, while the other methods appear to be barely perturbed. In fact, even though the other methods appear to be returning nearly perfect results. 


\section{ACKNOWLEDGMENT}

The authors would like to thank Mr. Ankush Kansal for willingly answering numerous questions regarding the Video algorithms. The authors would also like to thank Mr. Parminder Singh Reel for many valuable discussions; and the authors of the edge detectors for making the implementations available. Early results of this work appear in [8], [9],[17].

\section{REFERENCES}

[1] Rafael C. Gonzalez, Richard E. Woods, Digital Image Processing, Beijing: Publishing House of Electronics Industry, $2^{\text {nd }}$ Edition 2003.

[2] Kenneth R. Castleman, Digital Image Processing, Pearson Education Asia Limited and Tsinghua University Press, 2003.

[3] J. Batlle, J. Marti, P. Ridao. "A New FPGA/DSP-Based Parallel Architecture for Real-time Image Processing", Real-Time Imaging, vol. 8, pp 345-356, 2002

[4] TMS320DM642 Video/ Imaging Fixed-Point Digital Signal Processor Data Manual[EB/OL].Texas Instruments Incorporated,2003.

[5] SMT339 User Manual (QCF42); Version 1.2, 8/11/00 Sundance Multiprocessor Technology Ltd. 1999.

[6] C.Qi, Y.H. Chen, T.S. Huang. The Real-time Image Processing Technique Based on DSP[J]. Wuhan University Journal of Natural Sciences, 2005,10(6):1025-1029 Daniel Baumgarthner, Peter Rossler and Wilfried Kubingger "performance Benchmark of DSP and FPGA Implementations of Low-Level Vision Algorithms," Computer Vision and Pattern Recogniton, 2007. CVPR '07. IEEE Conference, Digital Object Identifier, pp. 17-22, June 2007.

[7] Miguel A. Vega-Rodriguez, Juan M. Sanchez-Perez, Juan A. GomezPulido, "Real Time Image Processing with Reconfigurable Hardware", Electronics Circuits and Systems. The 8th IEEE International Conference on Digital Object Identifier, pp 213-216 Vol.1, Sept 2001.

[8] Jincheng Wu, Jingrui Sun, Wenying Liu, "Design and Implementation of Video Image edge Detection System Based on FPGA" 2010 3rd International Conference on Image and Signal Processing (CISP2010), 2010

[9] Duan Jinghong, Deng Yanling, Liang Kun "Development of Image Processing System Based on DSP and FPGA," Electronic Measurement and Instruments, 2007. ICEMI '07. 8th International Conference on Digital Object Identifier, vol. 2 pp. 791 -794, 18 July, 2007.

[10] C. Vivas, J. Gomez Ortega and M. Vargas "parallel DSP based implementation of an automated visual quality inspection system", Emerging Technologies and Factory Automation, 1999. proceedings. ETFA'99.199 7th IEEE International Conference, Digital Object Identifier: 10.1109/ ETFA. 1999.815429, pp.743-749 vol.1, Barcelona, Spain, 10/18/1999- 10/21/1999.

[11] Patrick Murphy, Vinay Bharadwaj, Erik Welsh, J.Patrick Frantz, "A DSP-Based Platform for Wireless Video Compression", Global Telecommunications Conference, 2002. GLOBE COM' 02.IEEE, Digital Object Identifier: 10.1109/ GLOCOM. 2002. 1188499, pp.1754-1758 vol.2, 17-21 Nov. 2002

[12] Manish Kumar Birla "FPGA Based Reconfigurable Platform for Complex Image Processing," Electro/information Technology, 2006 IEEE International Conference, Digital Object Identifier: 10.1109/EIT.2006.252111, pp. 204-209, East Lansing, MI, 7-10 May 2006.

[13] Miguel A. Vega-Rodriguez, Juan M. Sanchez-Perez, Juan A. GomezPulido "Real Time Image Processing with Reconfigurable Hardware", Electronics, Circuits and Systems, 2001. ICECS 2001. The 8th IEEE International Conference, Digital Object Identifier 10.1109/ ICECS. 2001. 957718, pp.213-216 vol.1, 2-5 Sept. 2001

[14] Daniel Baumgarthner, Peter Rossler and Wilfried Kubingger "performance Benchmark of DSP and FPGA Implementations of LowLevel VisionAlgorithms," Computer Vision and Pattern Recogniton, 2007. CVPR '07. IEEE Conference, Digital Object Identifier: 10.1109/ CVPR. 2007. 38342, pp. 1-8,June 2007.

[15] Duan Jinghong, Deng Yanling, Liang Kun "Development of Image Processing System Based on DSP and FPGA," Electronic Measurement and Instruments, 2007. ICEMI '07. 8th International Conference, Digital Object Identifier ICEMI..4350799, pp. 2-791 - 2-794, July 182007.

[16] Luxin Yan, Tianxu Zhang and Sheng Zhong "A DSP/FPGA-Based Parallel Architecture for Real-time Image Processing," Intelligent Control and Automation, 2006. WCICA 2006. The Sixth World Congress, Digital Object Identifier 10.1109/ WCICA.2006.1713959, pp.10022-10025, 21-23, June 2006.

[17] T. Peli and D. Malah, "A study of edge detection algorithms", IEEE transaction on Computer Graphics and Image Processing. vol. 20, no. 1, pp. 1-21, Sept. 1982.

[18] W. Lunscher and M. Beddoes, "Optimal edge detector evaluation", IEEE Transaction on System Manufacturing and Cybernetics, vol. SMC-16, pp. 304-312, Apr. 1986.

[19] Renyan Zhang, Guoliang Zhao and Li Su, "A New Edge Detection Method in Image Processing", Proceedings of ISCIT, pp 430-433, 2005.

[20] A. Rosenfeld and M. Thurston, "Edge and curve detection for visual scene analysis," IEEE Transaction On Computing, vol. C-20, no. 5, pp. $562-569,1971$.

[21] M. Boo, E. Antelo and J.D. Bruguera, "VLSI Implementation of an Edge Detector Based on Sobel Operator", Department of Electronics University of Santiago de Compostela Santiago de Compostela Spain, 1994.

[21] Yan Lei, Zho Gang, "The performance of image acquisition and processing system based on DSP-FPGA", International Conference on Smart Manufacturing And Application,2008 Korea.

[22] John Canny, "A Computational Approach to Edge detection", IEEE Transaction on Pattern Analysis and Machine Intelligence, vol-6, pp 679-698, 1986

[23] G.Padamvathi, P Subashini, "Performance evaluation of the various edge detectors and filters for the noisy IR images", IEEE proceedings on Sensors, Signals, Visualization, Imaging, Simulation And Materials, pp 199-203, 2009

[24] I.yasri, N.H hamid, "Performance analysis of FPGA based Sobel Edge Detection operator", International Conference on Electronic Design Malaysia,2008

[25] V. Ramesh , R. M. Haralick, "Performance characterization of edge detectors," SPIE Application and Artificial Intelligence and Machine Vision on Robotics, vol.1708, pp. 252-266, April. 1992.

[26] D. Marr and E. Hildreth, "Theory of Edge Detection", Proc. of the Royal Society of London B, vol. 207, pp. 187-217, 1980.

[27] W. Lunscher and M. Beddoes, "Optimal edge detector evaluation," IEEE Trans. Syst., Man, Cybern., vol. SMC-16, pp. 304-312, Apr. 1986.

[28] M.Shin,D. Goldgof, and K. W. Bowyer, "An objective comparison methodology of edge detection algorithms for structure from motion task," in Proc. IEEE Conf. Comput. Vision Pattern Recognit., Santa Barbara,CA, pp. 190-195, 1998.

[29] I. E. Abdou and W. K. Pratt, "Quantitative design and evaluation of enhancement/thresholding edge detectors," Proc. IEEE, vol. 67, pp. 753763, May 1979.

[30] G.B.Shaw, "Local and regional edge detectors: Some comparison", Computer Graphics an Image Processing, vol. 9, pp. 135-149, Feb. 1979.

[31] L. Rosenthaler, F.Heitger, O.Kubler, and R.von der Heydt, "Detection of general edges and key points," in Proceeding of ECCV, G.Sandini, pp. 78-86, 1992.

[32] F. Heijden, "Edge and line feature extraction based on covariance models IEEE Transaction on Pattern Analysis and Machine Intelligence, vol. 17, pp. 16-33, Jan. 1995.

[33] Huang Yu-cheng, Hu Guo-qing, Wu Xion-gying, "A Study of Denoising Methods in Face Recognition System[J].Control \& Automation, pp. 187-188, 2005.

[34] Xu Xian-ling, Lin Yi-shui, "Comparison and Analysis for Image Edge Detection Algorithms" [J]. Automation \& Information Engineering. pp. 44-46, 2007.

[35] S.P.Ierodiaconou, N, Dahnoun, L.-Q. Xu "Implementation and Optimisation of a Video Object Segmentation Algorithm on an Embedded DSP Platform," Crime and Security. 2006. The Institution of 
Engineering and Technology Conference, INSPEC Accession Number: 9518513, pp. 432-437, 13-14 June 2006.

\section{AUTHORS PROFILE}

Mandeep Kaur received the BTech. degree, in Electronics and Communication Engineering from Shaheed Bhagat Singh College of Engineering and Technology, Firozepur in 2008. She is currently pursuing the M.E degree in Electronics and Communication at Thapar University, Patiala.Her research interests include Video Compression and Evaluation of algorithms.
Dr. Kulbir Singh was born in Batala $(\mathrm{Pb})$ India. He received his BTech degree in 1997 from PTU, Jalandhar. He obtained his ME and PhD degree from Thapar Institute of Engineering and Technology, Patiala in 2000 and 2006 respectively. He worked as lecturer from 2000 to 2007 in Electronics and Communication Engineering Department, Thapar University, Patiala. Presently he is working as Assistant Professor in Thapar University, Patiala since July 2006. He has published about 60 research articles in refereed international journals, international conference and national conference. He is life time member of IETE and ISTE. His research interest include Digital Signal Processing, Image Processing, Fractional Fourier Transform and Communication systems. 